\title{
Effect of Pinching and Growth Regulators on Vegetative and Physiological Parameters
}

\author{
Ch. Mounika*, T. Suseela, A.V.D. Dorajeerao, P. Subbaramamma and R.V. Sujatha \\ College of Horticulture, Dr. Y.S.R Horticultural University Andhra Pradesh, India \\ *Corresponding author
}

\section{A B S T R A C T}

The present investigation entitled "Effect of pinching and growth regulators on vegetative and physiological parameters of chrysanthemum (Dendranthema morifolium) cv. Pusa

\begin{tabular}{|l|}
\hline Ke y w or d s \\
Chrysanthemum \\
$\begin{array}{l}\text { (Dendranthema } \\
\text { morifolium) } \text { cv. } \\
\text { Pusa Kesari }\end{array}$ \\
\hline Article Info \\
\hline $\begin{array}{l}\text { Accepted: } \\
15 \text { July } 2019 \\
\text { Available Online: } \\
10 \text { August } 2019\end{array}$ \\
\hline \hline
\end{tabular}
Kesari" was carried out at College of Horticulture, Venkataramannagudem, Dr. Y.S.R Horticultural University, during Kharif 2017. The experiment was conducted in Randomized Block Design with Fifteen treatment combinations comprising of 3 levels of pinching i.e. No pinching, Single pinching and Double pinching and 5 levels of growth regulators i.e., GA 3100 ppm, GA 3 @ 200 ppm, Triacontanol @ 50 ppm, Triacontanol @ $100 \mathrm{ppm}$ and Water spray) replicated thrice and the observations were recorded for vegetative and physiological parameters of chrysanthemum among all treatment combinations the maximum plant spread, number of leaves plant ${ }^{-1}$, leaf area plant ${ }^{-1}$, leaf area index, leaf area duration,, number of suckers plant ${ }^{-1}$, crop growth rate, relative growth rate, net assimilation rate, total chlorophyll content, fresh weight plant ${ }^{-1}$ and dry weight plant $^{-1}$ were recorded in double pinched plants sprayed with triacontanol @ $50 \mathrm{ppm}$. The maximum plant height and absolute growth rate was recorded in plants without pinching and sprayed with $\mathrm{GA}_{3} @ 200 \mathrm{ppm}$. Hence on the basis of results obtained from the present investigation it can be concluded that the double pinched plants sprayed with triacontanol @ $50 \mathrm{ppm}$ was found effective with respect to vegetative and physiological parameters.

\section{Introduction}

Chrysanthemum (chryos = golden; anthos $=$ flower) is a popular flower crop of commercial importance, which belongs to the family Asteraceae. The basic chromosomal number of the genus is 9 and a wide range of ploidy level is found in different cultivars of the species with $2 \mathrm{n}=36,45,47,51$ and 75 . It is commonly known as "Queen of the East", "Autumn Queen" and "Guldaudi". The chrysanthemums are now becoming popular due to impearled diversity in shape, size, colourful form of the florets and pretty foliage. Chrysanthemum is a qualitative short day plant with respect to flowering. Chrysanthemum flowers have both ray and disc florets, but the disc florets only can reproduce. The vase life of chrysanthemum is 7-14 days. Largest producers of cut chrysanthemum in the world are Netherland, Mexico, Italy, Spain, and Korea. Largest chrysanthemum producing states in India are Andhra Pradesh, Karnataka, Tamilnadu, West 
Bengal and Gujarath. In India, Chrysanthemum is cultivated in almost all the states with an estimated area of about 16,630 hectares with a total production of 17, $937 \mathrm{MT}$ as loose flowers and 5,720 MT as cut flowers. In India, Andhra Pradesh is the most prominent chrysanthemum growing state with an area of 6,140 hectares and with a production of 7,550 MT (NHB, 2017).

\section{Materials and Methods}

The experiment was conducted during kharif season of 2017 at college of horticulture, Dr. Y.S.R Horticultural University Andhra Pradesh, India. Thirty days old cuttings of chrysanthemum cv. Pusa Kesari were planted at a distance of $30 \mathrm{~cm} \times 30 \mathrm{~cm}$ in $2.1 \mathrm{~m} \mathrm{x} 2.1$ $\mathrm{m}$ size plots in the field. The experiment was laid out in a factorial randomized block design with 15 treatments $T_{1}$ (plants without pinching and sprayed with $\mathrm{GA}_{3} @ 200$ ppm), $\mathrm{T}_{2}$ (plants with single pinching and sprayed with $\mathrm{GA}_{3}$ @ $200 \mathrm{ppm}$ ), $\mathrm{T}_{3}$ (plants with double pinching and sprayed with $\mathrm{GA}_{3} @ 200$ ppm), $\mathrm{T}_{4}$ (plants without pinching and sprayed with $\mathrm{GA}_{3}$ @ $100 \mathrm{ppm}), \mathrm{T}_{5}$ (plants with single pinching and sprayed with $\mathrm{GA}_{3} @ 100$ ppm), $\mathrm{T}_{6}$ (plants with double pinching and sprayed with $\mathrm{GA}_{3} @$ $100 \mathrm{ppm}$ ), $\mathrm{T}_{7}$ (plants without pinching and sprayed with triacontanol @ 50 ppm), $\mathrm{T}_{8}$ (plants with single pinching and sprayed with triacontanol @ 50 ppm), $\mathrm{T}_{9}$ (plants with double pinching and sprayed with triacontanol @ $50 \mathrm{ppm}$ ), $\mathrm{T}_{10}$ (plants without pinching and sprayed with triacontanol @ 100 ppm), $\mathrm{T}_{11}$ (plants with single pinching and sprayed with triacontanol @100 ppm), $\mathrm{T}_{12}$ (plants with double pinching and sprayed with triacontanol @ $100 \mathrm{ppm}$ ), $\mathrm{T}_{13}$ (plants without pinching and sprayed with water), $\mathrm{T}_{14}$ (plants with single pinching and sprayed with water) and $\mathrm{T}_{15}$ (plants with double pinching and sprayed with water) with three replications. In case of no pinching the plants were not at all pinched out throughout the experimentation. In single pinching, the plants were pinched out by removal of the apical portion of the main stem at 20 DAP, whereas in double pinching the plants were pinched out by removal of the apical portion of the main stem at (20 DAP) and removal of the apical portion of the primary branches appeared after the first pinching at $(50 \mathrm{DAP})$ in the respective treatments. Spraying of growth regulators were done at 25 and 50 DAP. Field preparation, intercultural operations and irrigation were done as per the standard recommendations. The observations of vegetative growth characters and floral growth characters were recorded and analyzed statistically (CD at 5\% level) as procedure described by Panse and Sukhatme 1978.

\section{Results and Discussion}

It was observed that during all the stages of plant growth, plant height $(11.51,28.07,49.43$ and $55.94 \mathrm{~cm}$ at 30, 60, 90 and 120 DAP respectively) was recorded maximum in plants without pinching. The effect of pinching was supported by Khobragade et al., (2012) in China aster. Higher plant height was recorded in plants sprayed with $\mathrm{GA}_{3} @ 200$ ppm. It was observed that during all the stages of plant growth, plant height was maximum in plants without pinching and sprayed with $\mathrm{GA}_{3} @ 200$ ppm at 120 DAP $(58.94 \mathrm{~cm})$ and among the growth regulators $\mathrm{GA}_{3} @ 200$ ppm has recorded the maximum plant height (13.13, $29.09,50.21$ and $55.62 \mathrm{~cm}$ at 30, 60, 90 and 120 DAP respectively) due to an increase in cell elongation in apical meristem leading to an increase in intermodal length by creating more negative osmotic pressure as well as turgor pressure in the cells due to conversion of starch into sugar as it supported by Sajid et al., (2016) in chrysanthemum. In the present experiment the best combination of pinching and growth regulators was found to be the interaction of plants without pinching and were sprayed with $\mathrm{GA}_{3} @ 200$ ppm (14.84, 
32.63, 53.69, and $58.94 \mathrm{~cm}$ at 30, 60, 90 and 120 DAP respectively). So it is evident that for higher plant height we can go for plants without pinching along with $\mathrm{GA}_{3} @ 200$ ppm. Similar findings were earlier reported by Deshmukh et al., (2014) in African marigold, Sharma and Joshi (2015) in China aster, Sajid et al., (2016) in chrysanthemum (Table 1).

The maximum plant spread was recorded in double pinched plants $(7.83,13.72,27.08$ and $30.67 \mathrm{~cm}$ at 30, 60, 90 and 120 DAP respectively.) and it was on par with plant spread in plants $(7.81 \mathrm{~cm})$ with single pinching at 30 DAP. It might be due to pinching reduces the apical dominance and encourages the growth of lateral buds and plant spread. Plants sprayed with triacontanol @ 50 ppm has recorded the maximum plant spread (7.96, 12.93, 24.23 and $31.23 \mathrm{~cm}$ at 30, 60, 90 and 120 DAP, respectively) (Table 1), because of the positive role of triacontanol on plant spread due to its growth promoting action, through an enhanced photosynthetic activity and an increase in the availability of the food material to the meristamatic cells could be the reason for an increase in plant spread. The interaction effect of double pinched plants with combination of triacontanol @ 50 ppm recorded the maximum plant spread $(8.80 \mathrm{~cm}, 16.17 \mathrm{~cm}, 30.76 \mathrm{~cm}$ and $34.56 \mathrm{~cm}$ at 30, 60, 90 and 120 DAP, respectively). Thus the plant spread increased substantially by double pinching and triacontanol@50 ppm. The present findings are in line with the earlier findings of Patel (1995) in marigold, Kesav (2014) in marigold and Salve et al., (2016) in chrysanthemum.

The number of leaves plant ${ }^{-1}$ were maximum in double pinched plants $(27.83,76.50,106.0$ and 94.95 at 30, 60, 90 and 120 DAP respectively) (Table 1), This might be due to removal of apical dominance through pinching, it reduces the plant height and encourages the development of lateral branches, which might have favoured the production of more number of leaves plant $^{-1}$ (Salve et al., 2016). Foliar spray of triacontanol@50 ppm has produced more number of leaves plant ${ }^{-1}$ due to enhanced photosynthetic activity in plants and thereby increased the supply of food material to leaf primordial which helps in development of more number of leaves plant ${ }^{-1}$. Among the interactions double pinched plants sprayed with triacontanol @ 50 ppm has recorded the maximum number of leaves plant ${ }^{-1}$ (32.26, 81.40, 113.2 and 99.96 at 30, 60, 90 and 120 DAP respectively). Similar findings were also reported by Khobragade et al., (2012) in China aster.

The leaf area plant ${ }^{-1}$ was gradually increased from 30 to 90 DAP and later it was decreased gradually. It could be due to decrease in production of new leaves by diversion of photoassimilates towards the development of flower buds and development of suckers beyond 90 DAP and also due to decrease in size of the leaves due to wilting and senescence of older leaves at 120 DAP. Maximum leaf area was recorded in double pinched plants $(221.52,464.13,1022.85$ and $817.71 \mathrm{~cm}^{2}$ at $30,60,90$ and 120 DAP respectively) (Table 1), among the different growth regulator sprays, application of triacontanol @50 ppm has recorded the maximum leaf area plant ${ }^{-1}(231.23,447.96$, 906.48 and $753.21 \mathrm{~cm}^{2}$ at $30,60,90$ and 120 DAP respectively). The interaction effect of plants with double pinching and sprayed with triacontanol @ 50 ppm has recorded the maximum leaf area plant $^{-1}$ (238.90, 481.08, 1063.30 and $846.40 \mathrm{~cm}^{2}$ at $30,60,90$ and 120 DAP respectively).

The leaf area index was gradually increased from 30 to 90 DAP, later it was decreased due to reduction in number of leaves (84.50) and leaf area $\left(753.21 \mathrm{~cm}^{2}\right)$ plant $^{-1}$ (Table 1), Among different levels of pinching, plants 
with double pinching has recorded the maximum leaf area index $(0.304,0.510,0.634$ and 0.575 at 30, 60, 90 and 120 DAP respectively). Among different growth regulators sprays, the plants sprayed with triacontanol @ 50 ppm showed significantly the maximum leaf area index $(0.260,0.471$, 0.588 and 0.561 at 30, 60, 90 and 120 DAP respectively). Plants with double pinching (at 20 and 50 DAP) and were sprayed with triacontanol @ 50 ppm has recorded the maximum leaf area index $(0.322,0.538,0.643$ and 0.587 at 30, 60, 90 and 120 DAP respectively). Intreraction effect of plants with double pinching (at 20 and 50 DAP) and were sprayed with triacontanol @ 50 ppm has recorded the maximum leaf area index $(0.322$, $0.538,0.643$ and 0.587 at $30,60,90$ and 120 DAP respectively). Based on the results obtained, it could be evident that the leaf area index was maximum in plants with double pinching and were sprayed with triacontanol @ $50 \mathrm{ppm}$ could be attributed to the production of more number of leaves (113.2) and leaf area $\left(1063.30 \mathrm{~cm}^{2}\right)$ plant $^{-1}$. The present results are in agreement with the earlier findings of Anuradha et al., (2017a) in marigold and Sahu et al., (2017) in capsicum and Shinde et al., (2010) in chrysanthemum.

Plants with double pinching has recorded the maximum fresh weight plant $^{-1}(20.75,69.02$, 157.89 and $135.62 \mathrm{~g}$ at $30,60,90$ and 120 DAP respectively) (Table 3 ). This might be due to an enhanced vegetative growth of plants in terms of number of primary branches plant $^{-1}$ (9.86), number of secondary branches plant $^{-1}$ (17.90), number of leaves (113.20) and leaf area plant ${ }^{-1}\left(1063.30 \mathrm{~cm}^{2}\right)$. The results are in conformity with the findings of Joshi et al., (2002) and Rathore (2011) in marigold. Among the growth regulators, foliar application triacontanol @ 50 ppm has recorded the maximum fresh weight plant $^{-1}$ (19.07, 63.56, 147.92 and $132.46 \mathrm{~g}$ at 30, 60, 90 and 120 DAP respectively), due to Foliar sprays of triacontanol @ 50 ppm has enhanced the fresh weight plant $^{-1}$ by an increase in photosynthetic activity, which results in an increased supply of food material to the meristamatic tissues helps in production of more vegetative growth parameters there by increased the fresh weight plant $^{-1}$. The interaction effect of plants with double pinching and were sprayed with triacontanol @ $50 \mathrm{ppm}$ has recorded the maximum fresh weight plant $^{-1}(22.86,73.08,162.61$ and $139.30 \mathrm{~g}$ at $30,60,90$ and $120 \mathrm{DAP}$ respectively). The results obtained in the present experiment are in close conformity with those of Arora and Khanna (1986) and also Bhati and Chitkara (1987) in marigold.

Plants with double pinching recorded the maximum dry weight plant $^{-1}(9.41,24.61$, 60.14 and $53.47 \mathrm{~g}$ at 30, 60, 90 and 120 DAP respectively) (Table 3). Foliar application of triacontanol @ 50 ppm has recorded the maximum dry weight plant ${ }^{-1}$ (9.93, 24.62, 58.14 and $53.56 \mathrm{~g}$ at 30, 60, 90 and 120 DAP respectively). Among the interaction effects plants with double pinching and sprayed with triacontanol @ 50 ppm has recorded the maximum dry weight plant ${ }^{-1}(10.96,27.50$, 63.23 and $56.33 \mathrm{~g}$ at 30, 60, 90 and 120 DAP respectively) because the dry weight plant $^{-1}$ was positively correlated with the fresh weight plant $^{-1}$. It was evident from the data that an increase in dry weight plant $^{-1}$, in plants with double pinching could be due to an enhanced vegetative growth of plants which resulted in the accumulation of more dry matter plant ${ }^{-1}$. The results are in line with the findings of Joshi et al., (2002) and Rathore (2011) in marigold. Foliar sprays of triacontanol @ 50 ppm has enhanced the photosynthesis activity which results in an increased supply of food material for the production of more number of branches, leaves and leaf area plant ${ }^{-1}$ there by increased the dry weight plant $^{-1}$. Similar results were found by Kesav (2014) in marigold. 
Table.1 Effect of pinching and growth regulators on growth attributes in chrysanthemum (Dendranthema morifolium) cv. Pusa Kesari

\begin{tabular}{|c|c|c|c|c|c|c|c|c|c|c|c|c|c|c|c|c|c|c|c|c|c|c|c|}
\hline \multirow[t]{2}{*}{ TCttt } & \multicolumn{4}{|c|}{ Plant height (cm) } & \multicolumn{4}{|c|}{ Plant spread (cm) } & \multicolumn{4}{|c|}{ Number of leaves } & \multicolumn{4}{|c|}{ Leaf area $\left(\mathrm{cm}^{2}\right)$} & \multicolumn{4}{|c|}{ Leaf area index } & \multicolumn{3}{|c|}{ Leaf area duration } \\
\hline & $\begin{array}{c}30 \\
\text { DAP }\end{array}$ & $\begin{array}{c}60 \\
\text { DAP }\end{array}$ & $\begin{array}{c}90 \\
\text { DAP }\end{array}$ & $\begin{array}{c}120 \\
\text { DAP }\end{array}$ & $\begin{array}{l}30 \\
\text { DAP }\end{array}$ & $\begin{array}{l}60 \\
\text { DAP }\end{array}$ & $\begin{array}{l}90 \\
\text { DAP }\end{array}$ & $\begin{array}{l}120 \\
\text { DAP }\end{array}$ & $\begin{array}{l}30 \\
\text { DAP }\end{array}$ & $\begin{array}{l}60 \\
\text { DAP }\end{array}$ & $\begin{array}{l}90 \\
\text { DAP }\end{array}$ & $\begin{array}{l}120 \\
\text { DAP }\end{array}$ & $\begin{array}{l}30 \\
\text { DAP }\end{array}$ & $\begin{array}{l}60 \\
\text { DAP }\end{array}$ & $\begin{array}{l}90 \\
\text { DAP }\end{array}$ & $\begin{array}{l}120 \\
\text { DAP }\end{array}$ & $\begin{array}{l}30 \\
\text { DAP }\end{array}$ & $\begin{array}{l}60 \\
\text { DAP }\end{array}$ & \begin{tabular}{|l|}
90 \\
DAP
\end{tabular} & \begin{tabular}{|l|}
120 \\
DAP
\end{tabular} & $\begin{array}{l}30-60 \\
\text { DAP }\end{array}$ & $\begin{array}{l}60-90 \\
\text { DAP }\end{array}$ & $\begin{array}{l}90- \\
120 \\
\text { DAP }\end{array}$ \\
\hline \multicolumn{24}{|l|}{ Pinching } \\
\hline $\mathbf{P}_{0}$ & 11.51 & 28.07 & 49.43 & 55.94 & 5.53 & 8.70 & 16.34 & 24.18 & 23.62 & 49.42 & 56.77 & 54.51 & 195.90 & 405.35 & 783.94 & 625.70 & 0.194 & 0.326 & 0.486 & 0.451 & 14.69 & 19.64 & 9.28 \\
\hline $\mathbf{P}_{1}$ & 10.11 & 26.52 & 47.13 & 52.25 & 7.81 & 11.77 & 21.78 & 27.61 & 27.13 & 68.35 & 89.21 & 84.73 & 220.71 & 426.74 & 839.21 & 728.71 & 0.230 & 0.485 & 0.548 & 0.522 & 15.51 & 20.22 & 13.48 \\
\hline $\mathbf{P}_{2}$ & 10.11 & 24.18 & 43.68 & 48.03 & 7.83 & 13.72 & 27.08 & 30.67 & 27.83 & 76.50 & 106.0 & 94.95 & 221.52 & 464.13 & 1022.85 & 817.71 & 0.304 & 0.510 & 0.634 & 0.575 & 17.04 & 25.78 & 16.21 \\
\hline SEm \pm & 0.195 & 0.283 & 0.329 & 0.329 & 0.071 & 0.207 & 0.254 & 0.301 & 0.080 & 0.396 & 0.423 & 0.268 & 0.387 & 0.386 & 2.247 & 1.142 & 0.001 & 0.001 & 0.001 & 0.002 & 0.216 & 0.076 & 0.186 \\
\hline CD at $5 \%$ & 0.583 & 0.847 & 0.986 & 1.176 & 0.269 & 0.621 & 0.762 & 1.903 & 0.234 & 1.86 & 1.265 & 0.804 & 1.126 & 1.123 & 6.543 & 3.325 & 0.003 & 0.003 & 0.003 & 0.006 & 0.648 & 0.228 & 0.560 \\
\hline \multicolumn{24}{|c|}{ Growth regulators } \\
\hline $\mathbf{G}_{1}$ & 11.56 & 27.57 & 48.75 & 53.69 & 6.48 & 10.51 & 20.83 & 25.06 & 24.76 & 62.84 & 80.31 & 74.25 & 206.95 & 424.03 & 870.32 & 708.69 & 0.236 & 0.426 & 0.541 & 0.527 & 15.54 & 21.03 & 12.41 \\
\hline $\mathbf{G}_{\mathbf{2}}$ & 13.13 & 29.90 & 50.21 & 55.62 & 7.28 & 11.94 & 21.23 & 27.39 & 26.62 & 64.67 & 83.96 & 76.99 & 209.53 & 434.72 & 880.22 & 724.34 & 0.242 & 0.436 & 0.545 & 0.531 & 15.75 & 22.76 & 13.34 \\
\hline $\mathbf{G}_{3}$ & 10.18 & 26.48 & 46.77 & 50.96 & 7.96 & 12.93 & 24.23 & 31.23 & 29.52 & 69.06 & 93.72 & 84.50 & 231.23 & 447.96 & 906.48 & 753.21 & 0.260 & 0.471 & 0.588 & 0.561 & 17.23 & 24.32 & 15.40 \\
\hline $\mathbf{G}_{4}$ & 9.23 & 24.21 & 44.91 & 49.20 & 7.55 & 11.70 & 22.48 & 28.53 & 27.64 & 66.38 & 90.22 & 82.18 & 216.74 & 442.54 & 897.63 & 737.76 & 0.248 & 0.449 & 0.572 & 0.536 & 16.09 & 23.63 & 13.81 \\
\hline $\mathbf{G}_{5}$ & 8.80 & 23.12 & 43.44 & 48.54 & 6.01 & 9.47 & 18.32 & 24.68 & 22.44 & 60.83 & 76.76 & 71.89 & 199.11 & 411.12 & 855.34 & 696.33 & 0.230 & 0.413 & 0.528 & 0.520 & 14.12 & 17.67 & 9.97 \\
\hline SEm \pm & 0.286 & 0.382 & 0.546 & 0.459 & 0.116 & 0.242 & 0.301 & 0.365 & 0.104 & 0.536 & 0.568 & 0.427 & 0.499 & 0.498 & 2.901 & 1.474 & 0.002 & 0.003 & 0.002 & 0.004 & 0.352 & 0.121 & 0.285 \\
\hline CD at $5 \%$ & 0.855 & 1.143 & 1.639 & 1.337 & 0.406 & 0.726 & 0.903 & 1.095 & 0.302 & 1.605 & 1.702 & 1.281 & 1.453 & 1.450 & 8.447 & 4.293 & 0.006 & 0.009 & 0.006 & 0.012 & 1.056 & 0.363 & 0.853 \\
\hline \multicolumn{24}{|l|}{ Interaction } \\
\hline $\mathbf{T}_{1}\left(\mathbf{P}_{0} \mathbf{G}_{1}\right)$ & 12.77 & 29.37 & 51.05 & 56.38 & 5.23 & 8.56 & 15.83 & 22.66 & 22.60 & 47.80 & 53.10 & 50.00 & 188.23 & 399.30 & 779.62 & 605.11 & 0.190 & 0.315 & 0.459 & 0.414 & 14.625 & 18.466 & 8.954 \\
\hline $\mathbf{T}_{\mathbf{2}}\left(\mathbf{P}_{1} \mathbf{G}_{\mathbf{1}}\right)$ & 10.95 & 28.26 & 48.43 & 54.92 & 7.10 & 10.73 & 20.66 & 25.73 & 25.80 & 66.40 & 84.93 & 80.60 & 215.56 & 416.28 & 829.98 & 716.83 & 0.221 & 0.478 & 0.544 & 0.518 & 15.270 & 20.210 & 12.793 \\
\hline $\mathbf{T}_{\mathbf{3}}\left(\mathbf{P}_{\mathbf{2}} \mathbf{G}_{\mathbf{1}}\right)$ & 10.98 & 25.10 & 45.76 & 49.78 & 7.12 & 12.23 & 25.40 & 28.41 & 25.90 & 74.33 & 102.9 & 92.16 & 217.08 & 456.51 & 1001.37 & 804.13 & 0.298 & 0.487 & 0.621 & 0.551 & 16.768 & 24.416 & 15.508 \\
\hline $\mathbf{T}_{\mathbf{4}}\left(\mathbf{P}_{0} \mathbf{G}_{2}\right)$ & 14.84 & 32.63 & 53.69 & 58.94 & 5.66 & 8.93 & 16.31 & 24.76 & 23.76 & 49.66 & 56.83 & 52.00 & 192.96 & 409.61 & 788.15 & 626.93 & 0.196 & 0.331 & 0.463 & 0.418 & 14.916 & 20.672 & 9.540 \\
\hline $\mathbf{T}_{5}\left(\mathbf{P}_{1} \mathbf{G}_{2}\right)$ & 12.27 & 29.53 & 50.66 & 56.30 & 8.10 & 12.13 & 22.76 & 27.29 & 27.43 & 68.33 & 88.76 & 84.31 & 219.03 & 428.95 & 843.38 & 729.93 & 0.225 & 0.484 & 0.547 & 0.523 & 15.328 & 21.311 & 14.030 \\
\hline $\mathbf{T}_{\mathbf{6}}\left(\mathbf{P}_{2} \mathbf{G}_{2}\right)$ & 12.29 & 27.56 & 46.28 & 51.61 & 8.10 & 13.76 & 26.63 & 30.12 & 27.67 & 76.03 & 106.3 & 94.66 & 216.60 & 465.61 & 1009.13 & 816.16 & 0.305 & 0.494 & 0.626 & 0.554 & 17.008 & 26.322 & 16.475 \\
\hline $\mathbf{T}_{7}\left(\mathbf{P}_{0} \mathbf{G}_{3}\right)$ & 10.76 & 27.40 & 49.53 & 53.86 & 6.33 & 9.36 & 18.56 & 26.36 & 26.40 & 52.10 & 69.30 & 62.83 & 218.33 & 416.56 & 798.07 & 659.95 & 0.203 & 0.346 & 0.538 & 0.515 & 15.477 & 21.527 & 11.677 \\
\hline $\mathbf{T}_{8}\left(\mathbf{P}_{1} \mathbf{G}_{3}\right)$ & 9.89 & 27.24 & 47.53 & 51.24 & 8.76 & 13.27 & 23.36 & 32.76 & 30.90 & 73.70 & 98.66 & 90.73 & 236.46 & 446.25 & 858.07 & 753.30 & 0.256 & 0.509 & 0.563 & 0.531 & 16.673 & 23.249 & 15.801 \\
\hline $\mathbf{T}_{\mathbf{9}}\left(\mathbf{P}_{2} \mathbf{G}_{3}\right)$ & 9.90 & 24.80 & 43.27 & 47.61 & 8.80 & 16.17 & 30.76 & 34.56 & 32.27 & 81.40 & 113.20 & 99.96 & 238.90 & 481.08 & 1063.30 & 846.40 & 0.322 & 0.538 & 0.643 & 0.587 & 19.557 & 28.184 & 18.744 \\
\hline $\mathbf{T}_{10}\left(\mathbf{P}_{0} \mathbf{G}_{4}\right)$ & 9.97 & 26.35 & 47.63 & 51.93 & 5.56 & 8.63 & 17.46 & 24.43 & 24.13 & 50.90 & 67.96 & 59.10 & 199.11 & 412.18 & 791.10 & 643.00 & 0.198 & 0.334 & 0.529 & 0.508 & 15.047 & 21.023 & 10.192 \\
\hline $\mathbf{T}_{11}\left(\mathbf{P}_{1} \mathbf{G}_{4}\right)$ & 8.88 & 24.03 & 45.50 & 50.63 & 8.53 & 12.69 & 22.43 & 28.90 & 28.83 & 69.70 & 92.70 & 89.70 & 224.55 & 439.18 & 849.72 & 740.53 & 0.237 & 0.498 & 0.558 & 0.528 & 16.024 & 22.315 & 14.249 \\
\hline $\mathbf{T}_{12}\left(\mathbf{P}_{2} \mathbf{G}_{4}\right)$ & 8.83 & 22.26 & 41.60 & 45.33 & 8.57 & 14.80 & 28.56 & 32.26 & 29.98 & 78.56 & 110.0 & 97.76 & 226.58 & 476.26 & 1052.09 & 829.76 & 0.310 & 0.516 & 0.629 & 0.561 & 17.220 & 27.569 & 17.010 \\
\hline $\mathbf{T}_{13}\left(\mathbf{P}_{0} \mathbf{G}_{5}\right)$ & 9.25 & 24.60 & 45.25 & 51.60 & 4.96 & 8.00 & 13.56 & 22.66 & 21.23 & 46.66 & 51.66 & 48.63 & 180.91 & 389.11 & 762.76 & 593.51 & 0.186 & 0.306 & 0.443 & 0.401 & 13.421 & 16.526 & 6.050 \\
\hline $\mathbf{T}_{14}\left(\mathbf{P}_{1} \mathbf{G}_{5}\right)$ & 8.58 & 23.58 & 43.55 & 48.20 & 6.53 & 9.94 & 19.66 & 23.40 & 22.73 & 63.63 & 81.03 & 78.33 & 207.95 & 403.06 & 814.92 & 703.40 & 0.215 & 0.456 & 0.531 & 0.513 & 14.273 & 14.042 & 10.544 \\
\hline $\mathbf{T}_{15}\left(\mathbf{P}_{2} \mathbf{G}_{5}\right)$ & 8.56 & 21.18 & 41.52 & 45.83 & 6.56 & 11.66 & 23.73 & 27.98 & 23.36 & 72.20 & 97.60 & 88.73 & 208.48 & 441.21 & 988.36 & 792.10 & 0.289 & 0.479 & 0.611 & 0.542 & 14.685 & 22.442 & 13.323 \\
\hline SEm \pm & 0.481 & 0.634 & 0.875 & 0.851 & 0.187 & 0.449 & 0.555 & 0.666 & 0.180 & 0.932 & 0.991 & 0.695 & 0.864 & 0.863 & 5.025 & 2.554 & 0.003 & 0.004 & 0.003 & 0.006 & 0.568 & 0.197 & 0.471 \\
\hline CD at $5 \%$ & 1.438 & 1.990 & $2 . .625$ & 2.553 & 0.675 & 1.347 & 1.665 & 1.998 & 0.523 & 2.791 & 2.967 & 2.085 & 2.517 & 2.512 & 14.631 & 7.436 & 0.009 & 0.012 & 0.009 & 0.018 & 1.704 & 0.591 & 1.413 \\
\hline
\end{tabular}


Table.2 Effect of pinching and growth regulators on physiological attributes in Chrysanthemum (Dendranthema morifolium) cv. Pusa Kesari

\begin{tabular}{|c|c|c|c|c|c|c|c|c|c|c|c|c|c|c|c|c|}
\hline & \multicolumn{3}{|c|}{ Absolute growth rate $\left(\mathrm{cm} \mathrm{d}^{-1}\right)$} & \multicolumn{3}{|c|}{ Crop growth rate $\left(\mathrm{g} \mathrm{m}^{-2} \mathrm{~d}^{-1}\right)$} & \multicolumn{3}{|c|}{$\begin{array}{l}\text { Relative growth rate }\left(\mathrm{mg} \mathrm{mg}^{-1}\right. \\
\left.\mathrm{d}^{-1}\right)\end{array}$} & \multicolumn{3}{|c|}{$\begin{array}{l}\text { Net assimilation rate }\left(\mathrm{mg} \mathrm{cm}^{-2}\right. \\
\left.\mathrm{d}^{-1}\right)\end{array}$} & \multicolumn{4}{|c|}{ Total chlorophyll (SPAD units) } \\
\hline & $30-60$ DAP & $\begin{array}{l}60-90 \\
\text { DAP }\end{array}$ & $\begin{array}{l}90-120 \\
\text { DAP }\end{array}$ & $\begin{array}{l}30-60 \\
\text { DAP }\end{array}$ & $\begin{array}{l}60-90 \\
\text { DAP }\end{array}$ & $\begin{array}{l}90-120 \\
\text { DAP }\end{array}$ & $\begin{array}{l}30-60 \\
\text { DAP }\end{array}$ & $\begin{array}{l}60-90 \\
\text { DAP }\end{array}$ & $\begin{array}{l}90-120 \\
\text { DAP }\end{array}$ & $\begin{array}{l}30-60 \\
\text { DAP }\end{array}$ & $\begin{array}{l}60-90 \\
\text { DAP }\end{array}$ & $\begin{array}{l}90-120 \\
\text { DAP }\end{array}$ & $30 \mathrm{DAP}$ & 60 DAP & 90 DAP & 120 DAP \\
\hline \multicolumn{17}{|l|}{ Pinching } \\
\hline $\mathbf{P}_{1}$ & 0.559 & 0.650 & 0.199 & 0.315 & 1.030 & -0.034 & 9.46 & 35.95 & -5.190 & 0.626 & 0.745 & -0.177 & 40.51 & 46.16 & 50.66 & 43.09 \\
\hline $\mathbf{P}_{2}$ & 0.552 & 0.637 & 0.180 & 0.364 & 1.107 & -0.037 & 10.83 & 42.67 & -6.912 & 0.679 & 0.823 & -0.192 & 45.01 & 49.88 & 55.08 & 45.36 \\
\hline $\mathbf{P}_{3}$ & 0.551 & 0.598 & 0.167 & 0.424 & 1.113 & -0.045 & 13.96 & 51.19 & -9.130 & 0.749 & 0.849 & -0.213 & 49.97 & 53.91 & 63.10 & 49.52 \\
\hline SEm \pm & 0.002 & 0.001 & 0.001 & 0.002 & 0.001 & 0.003 & 0.028 & 0.242 & 0.033 & 0.05 & 0.11 & 0.040 & 0.364 & 0.238 & 0.201 & 0.230 \\
\hline CD at $5 \%$ & 0.006 & 0.003 & 0.003 & 0.007 & 0.001 & 0.009 & 0.081 & 0.724 & 0.095 & 0.014 & 0.27 & 0.100 & 1.061 & 0.693 & 0.584 & 0.669 \\
\hline \multicolumn{17}{|c|}{ Growth regulators } \\
\hline $\mathbf{G}_{1}$ & 0.567 & 0.648 & 0.201 & 0.333 & 1.069 & -0.041 & 9.94 & 41.30 & -8.05 & 0.546 & 0.720 & -0.195 & 43.83 & 45.74 & 53.84 & 44.43 \\
\hline $\mathbf{G}_{2}$ & 0.583 & 0.666 & 0.218 & 0.352 & 1.079 & -0.038 & 11.38 & 43.36 & -7.06 & 0.574 & 0.859 & -0.207 & 45.46 & 48.68 & 56.14 & 46.15 \\
\hline $\mathbf{G}_{\mathbf{3}}$ & 0.557 & 0.634 & 0.186 & 0.417 & 1.117 & -0.036 & 13.34 & 46.91 & -4.68 & 0.867 & 0.928 & -0.242 & 50.15 & 56.44 & 61.36 & 49.74 \\
\hline $\mathbf{G}_{\mathbf{4}}$ & 0.545 & 0.612 & 0.152 & 0.408 & 1.116 & -0.037 & 12.65 & 45.04 & -5.56 & 0.823 & 0.877 & -0.228 & 47.24 & 51.78 & 58.47 & 47.73 \\
\hline $\mathbf{G}_{5}$ & 0.519 & 0.582 & 0.144 & 0.330 & 1.012 & -0.043 & 9.68 & 39.73 & -10.01 & 0.614 & 0.646 & -0.100 & 40.81 & 47.29 & 51.60 & 41.91 \\
\hline SEm \pm & 0.004 & 0.002 & 0.002 & 0.003 & 0.003 & -0.006 & 0.036 & 0.285 & 0.042 & 0.06 & 0.06 & 0.060 & 0.470 & 0.307 & 0.259 & 0.296 \\
\hline CD at $5 \%$ & 0.012 & 0.006 & 0.006 & 0.009 & 0.009 & -0.017 & 0.105 & 0.851 & 0.123 & 0.038 & 0.48 & 0.130 & 1.369 & 0.894 & 0.756 & 0.863 \\
\hline \multicolumn{17}{|l|}{ Interaction } \\
\hline $\mathbf{T}_{1}\left(\mathbf{P}_{\mathbf{0}} \mathbf{G}_{1}\right)$ & 0.571 & 0.673 & 0.211 & 0.274 & 1.019 & -0.036 & 8.21 & 34.42 & -6.32 & 0.507 & 0.706 & -0.185 & 39.33 & 40.76 & 48.66 & 41.48 \\
\hline $\mathbf{T}_{2}\left(\mathbf{P}_{1} \mathbf{G}_{1}\right)$ & 0.566 & 0.653 & 0.203 & 0.334 & 1.060 & -0.039 & 9.32 & 40.23 & -7.43 & 0.547 & 0.717 & -0.199 & 43.33 & 45.46 & 52.53 & 43.86 \\
\hline $\mathbf{T}_{3}\left(\mathbf{P}_{2} \mathbf{G}_{1}\right)$ & 0.564 & 0.619 & 0.191 & 0.393 & 1.130 & -0.048 & 12.31 & 49.25 & -10.42 & 0.585 & 0.738 & -0.201 & 48.83 & 51.00 & 60.33 & 47.95 \\
\hline $\mathbf{T}_{4}\left(\mathbf{P}_{0} \mathbf{G}_{2}\right)$ & 0.592 & 0.693 & 0.229 & 0.296 & 1.033 & -0.034 & 9.12 & 36.25 & -4.85 & 0.557 & 0.824 & -0.189 & 41.01 & 43.23 & 50.43 & 43.50 \\
\hline $\mathbf{T}_{5}\left(\mathbf{P}_{1} \mathbf{G}_{2}\right)$ & 0.579 & 0.673 & 0.219 & 0.349 & 1.068 & -0.037 & 11.42 & 42.62 & -6.74 & 0.560 & 0.865 & -0.207 & 45.35 & 48.50 & 54.90 & 45.76 \\
\hline $\mathbf{T}_{6}\left(\mathbf{P}_{2} \mathbf{G}_{2}\right)$ & 0.578 & 0.634 & 0.198 & 0.411 & 1.137 & -0.045 & 13.62 & 51.23 & -9.61 & 0.606 & 0.890 & -0.226 & 50.03 & 54.33 & 63.10 & 49.20 \\
\hline $\mathbf{T}_{7}\left(\mathbf{P}_{0} \mathbf{G}_{3}\right)$ & 0.561 & 0.654 & 0.198 & 0.371 & 1.053 & -0.032 & 11.20 & 39.25 & -2.75 & 0.792 & 0.856 & -0.217 & 43.54 & 53.03 & 54.80 & 46.40 \\
\hline $\mathbf{T}_{\mathbf{8}}\left(\mathbf{P}_{1} \mathbf{G}_{3}\right)$ & 0.557 & 0.649 & 0.185 & 0.409 & 1.108 & -0.035 & 12.31 & 46.84 & -4.52 & 0.870 & 0.937 & -0.235 & 48.90 & 56.53 & 60.66 & 49.54 \\
\hline $\mathbf{T}_{9}\left(\mathbf{P}_{2} \mathbf{G}_{3}\right)$ & 0.555 & 0.601 & 0.175 & 0.471 & 1.191 & -0.041 & 16.52 & 54.65 & -6.78 & 0.939 & 0.991 & -0.275 & 58.03 & 59.76 & 68.63 & 53.29 \\
\hline $\mathbf{T}_{10}\left(\mathbf{P}_{0} \mathbf{G}_{4}\right)$ & 0.549 & 0.627 & 0.177 & 0.365 & 1.506 & -0.033 & 10.53 & 37.32 & -3.25 & 0.789 & 0.846 & -0.206 & 42.00 & 49.26 & 52.60 & 44.56 \\
\hline $\mathbf{T}_{11}\left(\mathbf{P}_{1} \mathbf{G}_{4}\right)$ & 0.545 & 0.624 & 0.158 & 0.399 & 1.113 & -0.036 & 12.01 & 44.84 & -6.13 & 0.818 & 0.883 & -0.222 & 47.26 & 51.30 & 56.56 & 47.13 \\
\hline $\mathbf{T}_{12}\left(\mathbf{P}_{2} \mathbf{G}_{4}\right)$ & 0.543 & 0.586 & 0.141 & 0.461 & 1.179 & -0.042 & 15.41 & 52.98 & -7.32 & 0.862 & 0.902 & -0.256 & 52.46 & 54.80 & 66.26 & 51.52 \\
\hline $\mathbf{T}_{13}\left(\mathbf{P}_{0} \mathbf{G}_{5}\right)$ & 0.526 & 0.605 & 0.164 & 0.272 & 0.994 & -0.038 & 7.96 & 32.52 & -8.78 & 0.483 & 0.497 & -0.091 & 36.70 & 44.56 & 46.84 & 39.53 \\
\hline $\mathbf{T}_{14}\left(\mathbf{P}_{1} \mathbf{G}_{5}\right)$ & 0.516 & 0.588 & 0.138 & 0.332 & 1.006 & -0.041 & 9.12 & 38.84 & -9.74 & 0.603 & 0.716 & -0.101 & 40.23 & 47.63 & 50.76 & 40.55 \\
\hline $\mathbf{T}_{15}\left(\mathbf{P}_{2} \mathbf{G}_{5}\right)$ & 0.515 & 0.554 & 0.131 & 0.388 & 1.037 & -0.050 & 11.98 & 47.84 & -11.52 & 0.755 & 0.725 & -0.110 & 45.50 & 49.70 & 57.20 & 45.66 \\
\hline SEm \pm & 0.006 & 0.003 & 0.003 & 0.005 & 0.004 & 0.009 & 0.062 & 0.285 & 0.073 & 0.110 & 0.170 & 0.100 & 0.814 & 0.532 & 0.449 & 0.513 \\
\hline CD at $5 \%$ & 0.018 & 0.009 & 0.009 & 0.015 & 0.010 & 0.026 & 0.182 & 0.851 & 0.213 & 0.052 & 0.750 & 0.230 & 2.371 & 1.549 & 1.307 & 1.495 \\
\hline
\end{tabular}


Int.J.Curr.Microbiol.App.Sci (2019) 8(8): 2031-2042

Table.3 Effect of pinching and plant growth regulators on fresh weight, dry weight and number of suckers

\begin{tabular}{|c|c|c|c|c|c|c|c|c|c|c|}
\hline & \multicolumn{4}{|c|}{ Fresh weight } & \multicolumn{4}{|c|}{ Dry weight } & \multicolumn{2}{|c|}{$\begin{array}{c}\text { Number of } \\
\text { suckers }\end{array}$} \\
\hline & $\begin{array}{c}30 \\
\text { DAP }\end{array}$ & $\begin{array}{c}60 \\
\text { DAP }\end{array}$ & $\begin{array}{c}90 \\
\text { DAP }\end{array}$ & $\begin{array}{c}120 \\
\text { DAP }\end{array}$ & $\begin{array}{c}30 \\
\text { DAP }\end{array}$ & $\begin{array}{c}60 \\
\text { DAP }\end{array}$ & $\begin{array}{c}90 \\
\text { DAP }\end{array}$ & $\begin{array}{c}120 \\
\text { DAP }\end{array}$ & $\begin{array}{c}90 \\
\text { DAP }\end{array}$ & $\begin{array}{l}120 \\
\text { DAF }\end{array}$ \\
\hline $\mathbf{P}_{0}$ & 14.92 & 53.82 & 136.14 & 125.07 & 7.02 & 19.08 & 50.80 & 47.16 & 1.86 & 4.26 \\
\hline $\mathbf{P}_{1}$ & 17.51 & 60.47 & 141.22 & 128.36 & 7.83 & 21.22 & 55.06 & 50.68 & 3.46 & 6.40 \\
\hline $\mathbf{P}_{2}$ & 20.75 & 69.02 & 157.89 & 135.62 & 9.41 & 24.61 & 60.14 & 53.47 & 4.53 & 7.93 \\
\hline SEm \pm & 0.053 & 0.249 & 0.290 & 0.269 & 0.020 & 0.021 & 0.105 & 0.195 & 0.165 & 0.117 \\
\hline CD at 5\% & 0.153 & 0.725 & 0.844 & 0.808 & 0.059 & 0.062 & 0.306 & .587 & 0.480 & 0.340 \\
\hline $\mathbf{G}_{1}$ & 17.34 & 59.46 & 143.74 & 128.56 & 7.09 & 20.40 & 54.19 & 49.28 & 3.33 & 6.11 \\
\hline $\mathbf{G}_{\mathbf{2}}$ & 17.55 & 60.78 & 144.42 & 129.46 & 7.95 & 21.55 & 55.45 & 0.18 & 5.66 & 10.77 \\
\hline $\mathbf{G}_{\mathbf{3}}$ & 19.07 & 63.56 & 147.92 & 132.10 & 9.93 & 24.62 & 58.14 & 53.56 & 2.77 & 5.11 \\
\hline $\mathbf{G}_{4}$ & 18.42 & 61.11 & 145.99 & 130.61 & 8.63 & 22.72 & 56.81 & 51.92 & 2.44 & 4.8 \\
\hline $\mathbf{G}_{5}$ & 16.77 & 59.89 & 143.36 & 127.67 & 6.84 & 18.90 & 52.07 & 47.25 & 2.22 & 4.11 \\
\hline SEm \pm & 0.068 & 0.321 & 0.374 & 0.366 & 0.026 & 0.027 & 0.136 & 0.236 & 0.213 & 0.151 \\
\hline CD at $5 \%$ & 0.198 & 0.936 & 1.089 & 1.036 & 0.077 & 0.080 & 0.396 & 0.706 & 0.620 & 0.439 \\
\hline $\mathbf{T}_{\mathbf{1}}\left(\mathbf{P}_{\mathbf{0}} \mathbf{G}_{1}\right)$ & 14.98 & 53.73 & 134.90 & 24.80 & 6.14 & 18.04 & 49.50 & 6.13 & 2.33 & 5.00 \\
\hline $\mathbf{T}_{2}\left(\mathbf{P}_{1} \mathbf{G}_{1}\right)$ & 17.01 & 57.11 & 140.50 & 127.62 & 7.13 & 20.04 & 53.65 & 49.45 & 2.66 & 5.67 \\
\hline $\mathbf{T}_{\mathbf{3}}\left(\mathbf{P}_{\mathbf{2}} \mathbf{G}_{1}\right)$ & 20.03 & 68.56 & 155.82 & 133.26 & 8.02 & 23.13 & 59.42 & 52.27 & 5.00 & 7.66 \\
\hline $\mathbf{T}_{4}\left(\mathbf{P}_{0} \mathbf{G}_{2}\right)$ & 15.14 & 54.15 & 136.20 & 125.45 & 6.98 & 18.86 & 51.15 & 47.12 & 3.66 & 8.00 \\
\hline $\mathbf{T}_{5}\left(\mathbf{P}_{\mathbf{1}} \mathbf{G}_{2}\right)$ & 17.28 & 59.18 & 141.07 & 128.75 & 7.95 & 20.93 & 55.17 & 50.27 & 6.00 & 11.00 \\
\hline $\mathbf{T}_{\mathbf{6}}\left(\mathbf{P}_{2} \mathbf{G}_{2}\right)$ & 20.25 & 69.03 & 156.01 & 134.20 & 8.94 & 24.86 & 60.03 & 53.16 & 7.33 & 13.33 \\
\hline $\mathbf{T}_{7}\left(\mathbf{P}_{\mathbf{0}} \mathbf{G}_{3}\right)$ & 15.77 & 55.60 & 138.00 & 126.51 & 8.84 & 21.86 & 53.45 & 50.48 & 1.66 & 3.00 \\
\hline $\mathbf{T}_{8}\left(\mathbf{P}_{1} \mathbf{G}_{3}\right)$ & 18.58 & 62.00 & 143.17 & 130.50 & 9.99 & 24.50 & 57.75 & 53.88 & 3.66 & 5.66 \\
\hline $\mathbf{T}_{9}\left(\mathbf{P}_{2} \mathbf{G}_{3}\right)$ & 22.86 & 73.08 & 162.61 & 139.30 & 10.96 & 27.50 & 63.23 & 56.33 & 3.00 & 6.67 \\
\hline $\mathbf{T}_{10}\left(\mathbf{P}_{0} \mathbf{G}_{4}\right)$ & 15.53 & 54.43 & 137.70 & 125.96 & 7.92 & 20.12 & 52.16 & 48.84 & 0.66 & 3.00 \\
\hline $\mathbf{T}_{11}\left(\mathbf{P}_{1} \mathbf{G}_{4}\right)$ & 18.13 & 61.46 & 142.11 & 129.13 & 8.05 & 22.03 & 56.94 & 51.98 & 2.66 & 5.33 \\
\hline $\mathbf{T}_{12}\left(\mathbf{P}_{2} \mathbf{G}_{4}\right)$ & 21.61 & 70.46 & 158.18 & 136.76 & 9.94 & 26.02 & 61.35 & 54.95 & 4.00 & 6.33 \\
\hline $\mathbf{T}_{13}\left(\mathbf{P}_{0} \mathbf{G}_{5}\right)$ & 13.19 & 51.22 & 133.90 & 122.63 & 5.26 & 16.53 & 47.74 & 43.27 & 1.00 & 2.33 \\
\hline $\mathbf{T}_{14}\left(\mathbf{P}_{\mathbf{1}} \mathbf{G}_{5}\right)$ & 16.58 & 60.61 & 139.26 & 125.84 & 6.06 & 18.62 & 51.81 & 47.82 & 2.33 & 4.33 \\
\hline $\mathbf{T}_{15}\left(\mathbf{P}_{2} \mathbf{G}_{5}\right)$ & 19.04 & 67.86 & 156.87 & 134.62 & 9.22 & 21.55 & 56.66 & 50.66 & 3.33 & 5.67 \\
\hline SEm \pm & 0.118 & 0.557 & 648 & 0.635 & 0.046 & 0.047 & 0.235 & 0.431 & 0.369 & 0.261 \\
\hline Ce & 0.343 & 1.621 & 1.886 & 1.904 & 0.133 & 0.138 & 0.685 & 1.293 & 1.074 & 0.761 \\
\hline
\end{tabular}

The maximum number of suckers plant ${ }^{-1}$ were registered in plants with double pinching (4.53 and 7.93 at 90 and 120 DAP respectively) (Table 3). Among the growth regulators $\mathrm{GA}_{3} @ 200$ ppm has recorded the maximum number of suckers plant ${ }^{-1}(5.66$ and 10.77 at 90 and 120 DAP respectively). The interaction effects the double pinched plants 
sprayed with $\mathrm{GA}_{3} @ 200$ ppm has recorded the maximum number of suckers plant ${ }^{-1}(7.33$ and 13.33 at 90 and 120 DAP respectively). This might be due to plants with double pinching (at 20 and 50 DAP) and were sprayed with $\mathrm{GA}_{3} @ 200$ ppm as compared to the plants without pinching and were sprayed with water, might be due to an increase in number and size of leaves as well as shoot, results in translocation of higher photosynthates and eventually that would have been used for the production of propagules (suckers) (Sharifuzzaman et al., 2011). Similar results were also reported by sajid et al., (2016) in chrysanthemum.

The absolute growth rate was gradually increased from 30-60 DAP to 90-120 DAP later it was drastically reduced at 90-120 DAP due to slow increase in plant height. Among different pinching levels, plants without pinching recorded the maximum absolute growth rate $\left(0.559,0.650\right.$ and $0.199 \mathrm{~cm} \mathrm{~d}^{-1}$ at all dates of observations recorded) (Table 2). Among different growth regulators, foliar application of $\mathrm{GA}_{3} @ 200$ ppm has recorded the maximum absolute growth rate $(0.583$, 0.666 and $0.218 \mathrm{~cm} \mathrm{~d}^{-1}$ at $30-60,60-90$ and 90-120 DAP respectively). The interaction effect of plants without pinching and were sprayed with $\mathrm{GA}_{3} @ 200$ ppm recorded the maximum absolute growth rate $(0.592,0.693$ and $0.229 \mathrm{~cm} \mathrm{~d}^{-1}$ at $30-60,60-90$ and $90-120$ DAP respectively). The reason might be due to translocation of more photoassimilates towards the structural building of the plants during the pre-flowering stage. The increase in plant height was less during reproductive stage i.e., At 60 DAP due to diversion of more photoassimilates towards the initiation of inflorescence meristems (Vanitha Rani, 2016) and development of suckers at 90 DAP. Plants without pinching possess the apical dominance due to production of more auxins endogenously which lead to the more plant height, as compared to the plants with single pinching at (20 DAP) and with double pinching (20 and 50 DAP) levels. Plants sprayed with $\mathrm{GA}_{3}$ recorded the maximum absolute growth rate due to an increase in cell elongation in the apical meristem might be the reason to produce more absolute growth rate. Similar findings were reported by Vanitharani (2016) in chrysanthemum.

The crop growth rate was gradually increased from 30 to 90 DAP, later it was gradually decreased due to decrease in dry weight plant ${ }^{1}$ at 120 DAP. Plants with double pinching has recorded the maximum crop growth rate $\left(0.424,1.113\right.$, and $-0.452 \mathrm{~g} \mathrm{~m}^{-2} \mathrm{~d}^{-1}$ at all days of observations recorded) (Table 2). Among the growth regulators foliar application of triacontanol@50 ppm has recorded the maximum crop growth rate $(0.417,1.117$ and $-0.036 \mathrm{~g} \mathrm{~m}^{-2} \mathrm{~d}^{-1}$ at $30-60,60-90$ and $90-120$ DAP respectively). The interaction of plants with double pinching and were sprayed with triacontanol @ 50 ppm has recorded the maximum crop growth rate $(0.471,1.191$ and $-0.041 \mathrm{~g} \mathrm{~m}^{-2} \mathrm{~d}^{-1}$ at $30-60,60-90$ and $90-120$ DAP respectively). It might be due to production of more number of primary branches on mainstem, secondary branches on primary branches, more number of leaves on branches and dry weight plant ${ }^{-1}$ upto 90 DAP, later it was decreased due to decrease in production of branches and leaves on branches, senescence and abscission of older leaves and an increase in production of number of suckers plant ${ }^{-1}$. The results are in line with the findings of Dorajeerao and Mokashi (2012b) in annual chrysanthemum, Aparna (2012) in chrysanthemum and Vanitha Rani (2016) in chrysanthemum.

Among the Pinching levels, plants with double pinching has recorded the maximum (13.96, 51.19 and $-9.13 \mathrm{mg} \mathrm{mg}^{-1} \mathrm{~d}^{-1}$ at 30-60, 60-90 and 90-120 DAP respectively) relative growth rate. Among the growth regulators the maximum relative growth rate was noticed in 
plants sprayed with triacontanol @ 50 ppm (13.34, 46.91 and $-4.98 \mathrm{mg} \mathrm{mg}^{-1} \mathrm{~d}^{-1}$ at 30-60, 60-90 and 90-120 DAP respectively) (Table $2)$. The interaction effect of plants with double pinching and were sprayed with triacontanol @ 50 ppm recorded the maximum relative growth rate $(16.52,54.65$ and $-6.78 \mathrm{mg} \mathrm{mg}^{-1} \mathrm{~d}^{-1}$ at 30-60, 60-90 and 90120 DAP respectively). Based on the data it was evident that plants with double pinching and were sprayed with triacontanol @ 50 ppm has increased the relative growth rate from 30-90 DAP. Thereafter, the relative growth rate was found to be declined and became negative. Relative growth rate pointed towards the rate of dry matter production per unit of dry matter already existing in the plant. The relative growth rate was found to be increased from 30-90 days after transplanting and there after it was decreased due to an increase in the formation of more number of structural tissues than to the photosynthetic tissues. Further, it was observed that with the passage of time the plant dry weight was decreased due to translocation of photoassimilates to the reproductive organs than to structural organ development. Further, a drastic decline in the rate of photosynthesis due to senescence of older leaves and lack of availability of solar radiation to the leaves originated in the lower strata of the canopy due to the smothering of the crop during the full flowering stage. The results are in line with the findings of Aparna (2012) in chrysanthemum, shinde (2010) in chrysanthemum and Vanitha Rani (2016) in chrysanthemum.

The total chlorophyll content was gradually increased from 30 to 90 DAP later it was decreased at 120 DAP. It could be due to reduction in chlorophyll content due to degradation of chlorophyll by activation of hydrolytic enzymes as the leaf enters into senescence stage. Among all levels of pinching, plants with double pinching has recorded the maximum total chlorophyll content (49.97, 53.91, 63.10 and 49.52 SPAD units at 30,60, 90 and 120 DAP respectively) (Table 2). Among the growth regulators, plants sprayed with triacontanol @ 50 ppm has recorded the maximum total chlorophyll content $(50.15,56.44,61.36$ and 49.74 SPAD units at 30, 60, 90 and 120 DAP respectively). The interaction effect of double pinched plants sprayed with triacontanol @ 50 ppm has recorded the maximum total chlorophyll content. This is due to The increase in chlorophyll content in double pinched plants might be due to positive interaction of carboxylation efficiency and greater reduction in photooxidation rates leading to the development of more chlorophyll content. Further, the plants sprayed with triacontanol @ 50 ppm has improved the total chlorophyll content in leaves in terms of activation of the rubisco enzyme and stimulated the photosystem II activity (Verma et al., (2009); Shahbaz et al., (2013); Aziz et al., (2013) and thus improved the general health of plants. Similar results were reported by Rathore et al., (2011) in marigold, Aparna (2012) in chrysanthemum, Kesav (2014) in African marigold, and Vijayakumar (2017) in China aster.

The net assimilation rate was gradually increased 30-90 days after transplanting. After that NAR was declined and became negative in magnitude. Among the different pinching levels plants with double pinching has recorded the maximum net assimilation rate $\left(0.749 \mathrm{mg} \mathrm{cm}^{-2} \mathrm{~d}^{-1}, 0.849 \mathrm{mg} \mathrm{cm}^{-2} \mathrm{~d}^{-1}\right.$ and $0.213 \mathrm{mg} \mathrm{cm}^{-2} \mathrm{~d}^{-1}$ at $30-60,60-90$ and $90-120$ DAP respectively) (Table 2). Among the foliar application of growth regulators plants sprayed with triacontanol @ 50 ppm has recorded the maximum net assimilation rate (0.867, 0.928 and $\left.-0.242 \mathrm{mg} \mathrm{cm}^{-2} \mathrm{~d}^{-1}\right)$. Among the interaction effects double pinched plants sprayed with triacontanol @ 50 ppm has recorded the maximum net assimilation rate 
(0.939, 0.991 and $-0.275 \mathrm{mg} \mathrm{cm}^{-2} \mathrm{~d}^{-1}$ at 30-60, 60-90 and 90-120 DAP respectively). This might be due to the NAR was more in plants with double pinching and were sprayed with triacontanol @ 50 ppm could be attributed to an increase in number of primary and secondary branches plant ${ }^{-1}$, number of leaves, leaf area plant ${ }^{-1}$ and whole plant dry weight plant $^{-1}$. An increase in photosynthetic activity due to an increase in leaf area duration might helps in accumulation of more photoassimilates in the plant. Application of triacontanol@50 ppm has improved the Rubisco activity there by increased the photosynthetic activity and dry matter accumulation in the plant. The results are in line with the findings of Shinde (2010) in chrysanthemum, Dorajeerao and Mokashi (2012b) in annual chrysanthemum, Vanitharani (2016) in chrysanthemum and chandana (2017) in strawberry.

Leaf area duration was increased upto 90 DAP, later it was decreased due to diversion of hormones to the floral meristems instead of supply to the leaves during reproductive stage. The maximum leaf area duration (17.04, 25.78 and $16.21 \mathrm{~cm}^{2} \mathrm{~d}^{-1}$ at 30-60, 6090 and 90-120 DAP respectively) (Table 1), was recorded in plants with double pinching. Among the application of growth regulators maximum leaf area duration $(17.23,24.32$ and $15.40 \mathrm{~cm}^{2} \mathrm{~d}^{-1}$ at 30-60, 60-90 and 90-120 DAP respectively) recorded in plants sprayed with triacontanol @ 50 ppm. Interaction effect of double pinched plants sprayed with triacontanol @ 50 ppm has recorded the maximum leaf area duration $(19.557,28.184$ and $18.744 \mathrm{~cm}^{2} \mathrm{~d}^{-1}$ at $30-60,60-90$ and $90-$ 120 DAP respectively). The decrease in the number of leaves plant ${ }^{-1}$, leaf area index and leaf area duration was observed at 120 DAT. It could be due to lack of production functional leaves plant $^{-1}$, ageing and senescence of the matured leaves has been observed in all the treatments at 120 DAP.
The results are in line with the findings of Shinde (2010) in chrysanthemum, Dorajeerao and Mokashi (2012b) in annual chrysanthemum and Vanitha Rani (2016) in chrysanthemum.

Among the different treatment combinations, growth parameters viz., plant spread, number of leaves plant ${ }^{-1}$, leaf area plant $^{-1}$, leaf area index, leaf area duration, fresh weight plant ${ }^{-1}$ and dry weight plant $^{-1}$ were significantly superior in double pinched plants with foliar spray of triacontanol @ $50 \mathrm{ppm}$ in an increasing trend at 30,60 and 90 DAP but slight decline was observed at 120 DAP. The physiological parameters like crop growth rate, relative growth rate, net assimilation rate, total chlorophyll content were recorded maximum in double pinched plants sprayed with triacontanol @ 50 ppm in an increasing trend at 30, 60 and 90 DAP but slight decline was observed at 120 DAP.

\section{References}

Anuradha, $\quad$ R.W., $\quad$ Sateesh, $\quad$ R.P., Naveenakumar, Priyanka, T.K. and Kulakarni, B.S. 2017a. Effect of growth regulators and pinching on vegetative, flowering and flower yield parameters in african marigold cv. Calcutta Orange. International Journal of Pure and Applied Biosciences. 5 (5): 989-94.

Arora, J. S. and Khanna, K. 1986. Effect of nitrogen and pinching on growth and flower production of marigold (Tagetes erecta). Indian Journal of Horticulture. 43 (3/4): 291-94.

Bhati, R.S. and Chitkara, S.D. 1987. Effect of pinching and planting distance on the growth and yield of marigold (Tagetes erecta L.). Research and Development reporter. 4 (2): 159-64.

Chandana, A.N.V. 2017. Studies on the effect of plant growth regulators on plant growth, fruit set, yield and quality of 
strawberry (Fragaria ananassa Duch) cv. Camarosa in coastal Andhra Pradesh, M.Sc thesis, Dr. Y. S. R. Horticultural University Venkataramannagudem, West Godavari Dist.

Deshmukh, H.N., Ramdevputra, M.V. and Sahare, H.A. 2014. Effect of Spacing and gibberellic acid $\left(\mathrm{GA}_{3}\right)$ on growth parameters and yield of african mariglod (Tagetets erecta L.) cultivars. Trends in biosciences. 7 (22): 3622-627.

Kesav, C.R. 2014. Studies on effect of pinching and growth regulators and growth, flowering and yield of african marigold (Tagetus erecta L.) cv. Pusa Narangi Gainda, M.Sc thesis, Indira Gandhi krishi vishwa vidyalaya, Raipur. Khobragade, R.K., Bisen, S. and Thakur, R. S. 2012. Effect of planting distance and pinching on growth, flowering and yield of China aster (Callistephus chinensis) cv. Poornima. Indian Journal of Agricultural Sciences. 82 (4): 334-39.

NHB 2017. State wise area and production of loose flowers. Horticultural Statistics at a Glance 2016-17, National Horticulture Board, Ministry of Agriculture and Farmers welfare, Government of India, Gurgon. New Delhi. http://www.nhb.gov.in. pp. 222-23.

Panse, V.G. and Sukhatme, B.V. 1985. Statistical methods for agricultural workers. ICAR publication, New Delhi. 100-61.

Rathore, I., Mishra, A., Moond, S.K. and Bhatnagar, P. 2011. Studies on effect of pinching and plant bioregulators on growth and flowering of marigold (Tagetes erecta L.) cv. Pusa Basanti Gainda. Progressive Horticulture 43 (1): 52-55.

Sahu, G., Aslam, T., Das, S.P., Maity, T.K. and Gupta, N.K. 2017. A Study on preflowering foliar spray of plant growth regulator on growth and yield parameters in sweet pepper (Capsicum annuum L.) under protected condition. International Journal of Current Microbiology and Applied Sciences. 6 (7): 3998-4007.

Sajid, M., Amin, N., Ahmad, H. and Khan, K. 2016. Effect of gibberellic acid on enhancing flowering time in chrysanthemum (Chrysanthemum morifolium). 48 (2): 477-83.

Salve, D. M., Panchbhai, D.M., Badge, S. and Satar, V. 2016. Growth and flower yield of chrysanthemum as influenced by varieties and pinching. Plant Archives. 16 (2): 826-28.

Shahbaz, M. Noreen, N. and Perveen, S. 2013. Triacontanol modulates photosynthesis and osmoprotectants in canola (Brassica napus L.) under saline stress. Journal of Plant Interactions. 10: 764-69.

Sharifuzzaman, S.M., Ara, K.A., Rahman, M.H., Kabir, K. and Talukdar, M.B. 2011. Effect of $\mathrm{GA}_{3}, \mathrm{CCC}$, and $\mathrm{MH}$ on vegetative growth, flower yield and quality of chrysanthemum. Int. $j$. Expt.agric. 2 (1): 17-20.

Sharma, M.K. and Joshi, K. I. 2015. Effect of foliar spray of $\mathrm{GA}_{3}$ and NAA on growth flowering and yield of China aster (Callistephus chinensis NEES.) cultivars. International Journal of Agricultural Science and Research. 5 (4): 105-10.

Shinde, K.H. 2010. Investigation of different levels of gibberellic acid $\left(\mathrm{GA}_{3}\right)$ and pinching treatments on growth, flowering and yield of Chrysanthemum (Chrysanthemum morifolium R.) cv. IIHR-6 under middle Gujarat conditions. M.Sc. thesis. Anand Agricultural University, Gujarat.

Shinde, K.H. 2010. Investigation of different levels of gibberellic acid $\left(\mathrm{GA}_{3}\right)$ and pinching treatments on growth, flowering and yield of Chrysanthemum 
(Chrysanthemum morifolium R.) cv. IIHR-6 under middle Gujarat conditions. M.Sc. thesis. Anand Agricultural University, Gujarat.

Vanitarani, V. 2016. Studies on influence of nitrogen and potassium in combination with pinching on vegetative, physiological growth and flower yield attributes in chrysanthemum (Chrysanthemum morifolium), M.Sc thesis, Dr. Y. S. R. Horticultural University, Venkataramannagudem, Andhra Pradesh, India.

Verma, A., Malik, C.P., Sinsinwar, Y.K. and
Gupta, V.K. 2009. Yield parameters responses in a spreading $\mathrm{cv}$. M-13 and semi-spreading cv. Girnar-2 types of groundnut to six growth regulators. American-Eurasian Journal of Agricultural \& Environmental Sciences. 6: 88-91.

Vijayakumar, S., Rajadurai, K.R., Pandiyaraj, P. and Elangaivendan, A. 2017. Effect of plant growth regulators on vegetative and physiological parameters of China aster (Callistephus chinensis L. NEES.) $\mathrm{cv}$. Local. International Journal of Agriculture Sciences. 9 (17): 4148-150.

\section{How to cite this article:}

Mounika, Ch., T. Suseela, A.V.D. Dorajeerao, P. Subbaramamma and Sujatha, R.V. 2019. Effect of Pinching and Growth Regulators on Vegetative and Physiological Parameters. Int.J.Curr.Microbiol.App.Sci. 8(08): 2031-2042. doi: https://doi.org/10.20546/ijcmas.2019.808.237 